\title{
New non-overlapping domain decomposition methods for the harmonic Maxwell system
}

\author{
Ana Alonso Rodríguez * Luca Gerardo-Giorda ${ }^{\dagger}$
}

\begin{abstract}
We study a non-overlapping domain decomposition method for the harmonic Maxwell equations with a new kind of interface condition. We derive optimal transmission conditions for the harmonic Maxwell system in $\mathbb{R}^{3}$ following the procedure that M. Gander, F. Magoulès and F. Nataf have used for Helmholtz equation. We obtain in this way interface conditions with second order tangential derivatives, which guarantee convergence for both propagative and evanescent modes. We then propose two iterative solvers for the Maxwell equations based on a domain decomposition procedure where such conditions are enforced on the interface. Some numerical results for a two domain decomposition show the effectiveness of the optimized interface conditions.
\end{abstract}

\section{Introduction}

The numerical solution of the time-harmonic Maxwell system over a large three-dimensional domain is a difficult task. The linear system resulting from the finite element approximation is complex and the matrix is neither hermitian nor definite. In order to construct an efficient iterative method to solve this system, domain decomposition can be used. We concentrate in non overlapping domain decomposition methods. The idea is to decompose the domain into several non overlapping subdomains and to solve in parallel the local problems. This procedure leads to an iterative method that converges to the solution of the original problem if the solutions in the subdomains are related by means of suitable boundary conditions at the interface. The performance of the method depends drastically on the choice of this interface conditions.

The earliest non overlapping domain decomposition method for the time-harmonic Maxwell system was introduced in [5] with an interface condition of Robin type. For this algorithm it is proved that the local solutions converge to the corresponding restrictions of the global solution weakly in the spaces of complex vector functions of $L^{2}$ with curl in $L^{2}$. In his Ph.D. Thesis, Chevalier [2] proposed a modification of the Robin interface condition by adding a second order differential operator along the interface. For this new algorithm there is not an accurate convergence analysis. Another interface condition involving a second order tangential operator is analyzed in [3], where some numerical results are given for a model problem with conditions of radiative type on the boundary. A Fourier analysis shows that, for the interface conditions used in these three algorithms, the iteration map is not a contraction for evanescent modes.

\footnotetext{
${ }^{*}$ Dipartimento di Matematica, Università di Trento, Via Sommarive 14, I-38050 Povo (TN), Italy

${ }^{\dagger}$ CMAP, École Polytechnique, F-91128 Palaiseau Cedex, France
} 
Using this Fourier analysis and proceeding as M.J. Gander, F. Magoulès and F. Nataf for Helmholtz equation (see [6]) we identify two families of interface conditions that lead to iteration maps that are contractive for both propagative and evanescent modes. Both families involve a second order tangential differential operator. The first family of interface conditions depends on a complex parameter $p \in \mathbb{C}$, while the second one depends on two parameters, one real $(r \in \mathbb{R})$ and one purely imaginary $(i q, q \in \mathbb{R})$. We will refer in the sequel to the first family as to symmetric interface conditions, and to the second one as to unsymmetric interface conditions. The parameters $p \in \mathbb{C}$, and $r, q \in \mathbb{R}$ can then be optimized, namely they can be chosen in order to minimize the convergence rate of the corresponding iterative procedure.

The paper is organized as follows. In Section 2 the model problem in $\mathbb{R}^{3}$ is solved by means of an additive Schwarz method in a very general framework: by means of a Fourier analysis, we derive two families of interface conditions which guarantee convergence for both propagative and evanescent modes. An optimization is then performed on the parameters describing such families. In Section 3 the proposed domain decomposition algorithms are detailed for a two-domain decomposition at both the continuous and the discrete level. In Section 4 the algorithms are validated by some numerical tests. Finally, in Section 5, a survey of the non-overlapping domain decomposition algorithms for the harmonic Maxwell system previously appeared in literature is given: the interface conditions in these algorithms can be easily included in the general framework we introduced in Section 2, and are compared numerically with the conditions we propose.

\section{Optimized interface conditions for a model problem in $\mathbb{R}^{3}$}

The harmonic solutions of Maxwell equations are complex valued fields $\mathbf{E}$ and $\mathbf{H}$ such that the following fields

$$
\begin{aligned}
\mathcal{E}(\mathbf{x}, t) & =\mathcal{R} e(\mathbf{E}(\mathbf{x}) \exp (i \omega t)) \\
\mathcal{H}(\mathbf{x}, t) & =\mathcal{R} e(\mathbf{H}(\mathbf{x}) \exp (i \omega t))
\end{aligned}
$$

satisfy the Maxwell system. The positive number $\omega$ is called the pulsation of the harmonic wave. $\mathbf{E}$ and $\mathbf{H}$ satisfy the time-harmonic equations

$$
\begin{aligned}
& \operatorname{curl} \mathbf{E}-i \omega \mu \mathbf{H}=\mathbf{0} \\
& \operatorname{curl} \mathbf{H}+i \omega \varepsilon \mathbf{E}=\mathbf{J}
\end{aligned}
$$

where $\mathbf{J}$ is the amplitude of the applied current density.

The model problem we consider is the second order time-harmonic Maxwell equation obtained from (1) by eliminating either the magnetic field $\mathbf{H}$ or the electric filed $\mathbf{E}$. It reads

$$
\operatorname{curl} \operatorname{curl} \mathbf{u}-\omega^{2} \mathbf{u}=\mathbf{F} \quad \text { in } \Omega
$$

where, for sake of simplicity in notations, we have normalized both the electric permittivity $\varepsilon$ and the magnetic permeability $\mu$ (i.e. $\varepsilon=\mu=1$ ). When $\mathbf{u}$ stands for the electric field, $\mathbf{F}:=i \omega \mathbf{J}$; on the other hand, if $\mathbf{u}$ is the magnetic field, $\mathbf{F}:=\operatorname{curl} \mathbf{J}$.

We present the analysis in the domain $\Omega=\mathbb{R}^{3}$ with the radiation condition

$$
\lim _{r \rightarrow \infty} r(\operatorname{curl} \mathbf{u} \times \mathbf{n}+i \omega \mathbf{u})=\mathbf{0}
$$

where $r=|\mathbf{x}|, \mathbf{n}=\mathbf{x} /|\mathbf{x}|$. 
We decompose the domain in two non-overlapping subdomains $\Omega_{B}:=\mathbb{R}^{2} \times(-\infty, 0)$ and $\Omega_{T}:=\mathbb{R}^{2} \times(0,+\infty)$ and we set $\Gamma:=\overline{\Omega_{B}} \cap \overline{\Omega_{T}}$. Since, as it is well known in domain decomposition literature, an iterative algorithm of Schwarz type without overlap does not converge when Dirichlet interface conditions are used, we consider the following iterative algorithm with more general interface conditions: given $\mathbf{u}_{B}^{0}$ and $\mathbf{u}_{T}^{0}$, for $j \geq 0$ solve

$$
\begin{aligned}
& \begin{cases}\operatorname{curl} \operatorname{curl} \mathbf{u}_{B}^{j+1}-\omega^{2} \mathbf{u}_{B}^{j+1}=\mathbf{F} & \text { in } \Omega_{B} \\
\operatorname{curl} \mathbf{u}_{B}^{j+1} \times \mathbf{n}_{B}+S_{B}\left(\mathbf{u}_{B, \tau}^{j+1}\right)=\operatorname{curl} \mathbf{u}_{T}^{j} \times \mathbf{n}_{B}+S_{B}\left(\mathbf{u}_{T, \tau}^{j}\right) & \text { on } \Gamma,\end{cases} \\
& \begin{cases}\operatorname{curl} \operatorname{curl} \mathbf{u}_{T}^{j+1}-\omega^{2} \mathbf{u}_{T}^{j+1}=\mathbf{F} \\
\operatorname{curl} \mathbf{u}_{T}^{j+1} \times \mathbf{n}_{T}+S_{T}\left(\mathbf{u}_{T, \tau}^{j+1}\right)=\operatorname{curl} \mathbf{u}_{B}^{j} \times \mathbf{n}_{T}+S_{T}\left(\mathbf{u}_{B, \tau}^{j}\right) & \text { on } \Gamma .\end{cases}
\end{aligned}
$$

Here $S_{B}$ and $S_{T}$ are two linear operators acting in the tangential direction on the interface and $\mathbf{u}_{L, \tau}^{j}:=\mathbf{n}_{B} \times \mathbf{u}_{L}^{j} \times \mathbf{n}_{B}=\mathbf{n}_{T} \times \mathbf{u}_{L}^{j} \times \mathbf{n}_{T}$ for $L=B, T$. It is then easy to see that, if $\operatorname{ker}\left(S_{B}+S_{T}\right)=\{\mathbf{0}\}$, we recover at convergence the solution of the single domain problem.

Since the problems involved are linear, it is enough to analyze the convergence to the zero solution for the homogeneous system. Note that since curl curl $\mathbf{v}+\nabla \operatorname{div} \mathbf{v}=-\Delta \mathbf{v}$, the homogeneous equation curl curl $\mathbf{u}_{L}^{j+1}-\omega^{2} \mathbf{u}_{L}^{j+1}=\mathbf{0}$ is equivalent to

$$
\begin{array}{ll}
-\Delta \mathbf{u}_{L}^{j+1}-\omega^{2} \mathbf{u}_{L}^{j+1}=\mathbf{0} & \text { in } \Omega_{L} \\
\operatorname{div} \mathbf{u}_{L}^{j+1}=0 & \text { in } \Omega_{L} \quad L=B, T .
\end{array}
$$

We perform a partial Fourier transform in the $x$ and $y$ directions, that we denote with $\mathcal{F}$, and we call $k_{1}$ and $k_{2}$ the corresponding dual variables. The transform $\mathcal{F}$ is defined as

$$
\mathcal{F}: \mathbf{u}(x, y, z) \rightarrow \widehat{\mathbf{u}}\left(k_{1}, k_{2}, z\right)=\iint_{\mathbb{R}^{2}} \mathbf{u}(x, y, z) e^{-i\left(k_{1} x+k_{2} y\right)} d x d y
$$

Then we obtain

$$
\begin{aligned}
& \left\{\begin{array}{lr}
-\frac{\partial^{2} \widehat{\mathbf{u}}_{B}^{j+1}}{\partial z^{2}}+\left(k_{1}^{2}+k_{2}^{2}-\omega^{2}\right) \widehat{\mathbf{u}}_{B}^{j+1}=\mathbf{0} & \left(k_{1}, k_{2}\right) \in \mathbb{R}^{2}, z<0 \\
-i k_{1} \widehat{u}_{B, 1}^{j+1}-i k_{2} \widehat{u}_{B, 2}^{j+1}+\partial_{z} \widehat{u}_{B, 3}^{j+1}=0 & \left(k_{1}, k_{2}\right) \in \mathbb{R}^{2}, z<0 \\
\mathcal{F}\left(\operatorname{curl} \mathbf{u}_{B}^{j+1}\right) \times \mathbf{n}_{B}+\sigma_{B}\left(k_{1}, k_{2}\right)\left(\widehat{\mathbf{u}}_{B, \tau}^{j+1}\right) & \\
=\mathcal{F}\left(\operatorname{curl} \mathbf{u}_{T}^{j}\right) \times \mathbf{n}_{B}+\sigma_{B}\left(k_{1}, k_{2}\right)\left(\widehat{\mathbf{u}}_{T, \tau}^{j}\right) & \left(k_{1}, k_{2}\right) \in \mathbb{R}^{2}, z=0,
\end{array}\right. \\
& \left\{\begin{array}{lr}
-\frac{\partial^{2} \widehat{\mathbf{u}}_{T}^{j+1}}{\partial z^{2}}+\left(k_{1}^{2}+k_{2}^{2}-\omega^{2}\right) \widehat{\mathbf{u}}_{T}^{j+1}=\mathbf{0} & \left(k_{1}, k_{2}\right) \in \mathbb{R}^{2}, z>0 \\
-i k_{1} \widehat{u}_{T, 1}^{j+1}-i k_{2} \widehat{u}_{T, 2}^{j+1}+\partial_{z} \widehat{u}_{T, 3}^{j+1}=0 & \left(k_{1}, k_{2}\right) \in \mathbb{R}^{2}, z>0 \\
\mathcal{F}\left(\operatorname{curl} \mathbf{u}_{T}^{j+1}\right) \times \mathbf{n}_{T}+\sigma_{T}\left(k_{1}, k_{2}\right)\left(\widehat{\mathbf{u}}_{T, \tau}^{j+1}\right) & \\
=\mathcal{F}\left(\operatorname{curl} \mathbf{u}_{B}^{j}\right) \times \mathbf{n}_{T}+\sigma_{T}\left(k_{1}, k_{2}\right)\left(\widehat{\mathbf{u}}_{B, \tau}^{j}\right) & \left(k_{1}, k_{2}\right) \in \mathbb{R}^{2}, z=0,
\end{array}\right.
\end{aligned}
$$

where $\sigma_{L}$ denotes the symbol of the operator $S_{L}$. 
The general solutions of $-\frac{\partial^{2} \widehat{\mathbf{u}}_{L}}{\partial z^{2}}+\left(k_{1}^{2}+k_{2}^{2}-\omega^{2}\right) \widehat{\mathbf{u}}_{L}=\mathbf{0},(L=B, T)$ are

$$
\widehat{\mathbf{u}}_{L}\left(k_{1}, k_{2}, z\right)=\mathbf{a}_{L}\left(k_{1}, k_{2}\right) e^{\lambda z}+\mathbf{b}_{L}\left(k_{1}, k_{2}\right) e^{-\lambda z}
$$

where $\lambda=\lambda\left(k_{1}, k_{2}\right)=\sqrt{k_{1}^{2}+k_{2}^{2}-\omega^{2}}$ denotes the root of the characteristic equation which is either real and positive or purely imaginary. Since the radiation conditions exclude growing solutions as well as incoming modes at infinity we obtain that $\mathbf{b}_{B}\left(k_{1}, k_{2}\right)=\mathbf{a}_{T}\left(k_{1}, k_{2}\right)=\mathbf{0}$, hence

$$
\begin{aligned}
& \widehat{\mathbf{u}}_{B}\left(k_{1}, k_{2}, z\right)=\mathbf{a}_{B}\left(k_{1}, k_{2}\right) e^{\lambda z} \\
& \widehat{\mathbf{u}}_{T}\left(k_{1}, k_{2}, z\right)=\mathbf{b}_{T}\left(k_{1}, k_{2}\right) e^{-\lambda z} .
\end{aligned}
$$

From the transformed divergence free conditions $-i k_{1} \widehat{u}_{L, 1}-i k_{2} \widehat{u}_{L, 2}+\partial_{z} \widehat{u}_{L, 3}=0$ we get

$$
\begin{aligned}
\lambda \widehat{u}_{B, 3} & =\partial_{z} \widehat{u}_{B, 3}=i\left(k_{1} \widehat{u}_{B, 1}+k_{2} \widehat{u}_{B, 2}\right) \\
-\lambda \widehat{u}_{T, 3} & =\partial_{z} \widehat{u}_{T, 3}=i\left(k_{1} \widehat{u}_{T, 1}+k_{2} \widehat{u}_{T, 2}\right),
\end{aligned}
$$

thus, for $\lambda \neq 0$,

$$
\widehat{u}_{B, 3}=\frac{i}{\lambda}\left(k_{1} \widehat{u}_{B, 1}+k_{2} \widehat{u}_{B, 2}\right) \quad \widehat{u}_{T, 3}=-\frac{i}{\lambda}\left(k_{1} \widehat{u}_{T, 1}+k_{2} \widehat{u}_{T, 2}\right) .
$$

This means that we can write $\mathcal{F}\left(\operatorname{curl} \mathbf{u}_{L}\right) \times \mathbf{n}_{L}$ in terms of the tangential components on $\Gamma$, $\left(\widehat{u}_{L, 1}, \widehat{u}_{L, 2}\right)$. In fact we can prove the following result.

Lemma 1 Let $\mathbf{u}_{L}(L=B, T)$ be a solution of

$$
\begin{array}{ll}
-\Delta \mathbf{u}_{L}-\omega^{2} \mathbf{u}_{L}=\mathbf{0} & \text { in } \Omega_{L} \\
\operatorname{div} \mathbf{u}_{L}=0 & \text { in } \Omega_{L}, \\
\lim _{r \rightarrow \infty} r\left(\operatorname{curl} \mathbf{u}_{L} \times \mathbf{n}+i \omega \mathbf{u}_{L}\right)=0, &
\end{array}
$$

and let $\mathbf{U}_{L}:=\left[\begin{array}{l}\widehat{u}_{L, 1} \\ \widehat{u}_{L, 2}\end{array}\right]$. Then

$$
\lambda \mathcal{F}\left(\operatorname{curl} \mathbf{u}_{L}\right) \times \mathbf{n}_{L}=\left[\begin{array}{c}
R \mathbf{U}_{L}-\omega^{2} \mathbf{U}_{L} \\
\mathbf{0}
\end{array}\right]
$$

where $R=R\left(k_{1}, k_{2}\right)$ is the matrix

$$
R:=\left[\begin{array}{cc}
k_{2}^{2} & -k_{1} k_{2} \\
-k_{1} k_{2} & k_{1}^{2}
\end{array}\right] .
$$

Proof.- This result can be found in [2]. We include here the proof for completeness. We consider the case $L=B$ but the proof is analogous for $L=T$. Since

$$
\mathcal{F}\left(\operatorname{curl} \mathbf{u}_{B}\right)=\left[\begin{array}{c}
-i k_{2} \widehat{u}_{B, 3}-\frac{\partial \widehat{u}_{B, 2}}{\partial z} \\
\frac{\partial \widehat{u}_{B, 1}}{\partial z}+i k_{1} \widehat{u}_{B, 3} \\
-i k_{1} \widehat{u}_{B, 2}+i k_{2} \widehat{u}_{B, 1}
\end{array}\right]=\left[\begin{array}{c}
\frac{k_{2}}{\lambda}\left(k_{1} \widehat{u}_{B, 1}+k_{2} \widehat{u}_{B, 2}\right)-\lambda \widehat{u}_{B, 2} \\
\lambda \widehat{u}_{B, 1}-\frac{k_{1}}{\lambda}\left(k_{1} \widehat{u}_{B, 1}+k_{2} \widehat{u}_{B, 2}\right) \\
-i k_{1} \widehat{u}_{B, 2}+i k_{2} \widehat{u}_{B, 1}
\end{array}\right]
$$


we easily conclude

$\lambda \mathcal{F}\left(\operatorname{curl} \mathbf{u}_{B}\right) \times \mathbf{n}_{B}=\left[\begin{array}{c}\lambda^{2} \widehat{u}_{B, 1}-k_{1}\left(k_{1} \widehat{u}_{B, 1}+k_{2} \widehat{u}_{B, 2}\right) \\ -k_{2}\left(k_{1} \widehat{u}_{B, 1}+k_{2} \widehat{u}_{B, 2}\right)+\lambda^{2} \widehat{u}_{B, 2} \\ 0\end{array}\right]=\left[\begin{array}{c}\left(k_{2}^{2}-\omega^{2}\right) \widehat{u}_{B, 1}-k_{1} k_{2} \widehat{u}_{B, 2} \\ -k_{2} k_{1} \widehat{u}_{B, 1}+\left(k_{1}^{2}-\omega^{2}\right) \widehat{u}_{B, 2} \\ 0\end{array}\right]$

Remark 1 We recall that for a function $\mathbf{q}: \mathbb{R}^{2} \rightarrow \mathbb{R}^{2}, \operatorname{curl} \mathbf{q}=\frac{\partial q_{2}}{\partial x}-\frac{\partial q_{1}}{\partial y}$ and

$$
\overrightarrow{\operatorname{curl}} \operatorname{curl} \mathbf{q}=\left[\begin{array}{c}
\frac{\partial}{\partial y}\left(\frac{\partial q_{2}}{\partial x}-\frac{\partial q_{1}}{\partial y}\right) \\
-\frac{\partial}{\partial x}\left(\frac{\partial q_{2}}{\partial x}-\frac{\partial q_{1}}{\partial y}\right)
\end{array}\right]
$$

It is then easy to see that

$$
R \mathbf{U}_{L}=\mathcal{F}\left(\overrightarrow{\operatorname{curl}} \operatorname{curl} \mathbf{u}_{L, \tau}\right) .
$$

Since the third components in the above expressions vanish identically, we can express the action of one iteration of the algorithm (6), in terms of $\mathbf{U}_{B}$ and $\mathbf{U}_{T}$, as

$$
\begin{aligned}
& \frac{1}{\lambda}\left(R \mathbf{U}_{B}^{j+1}-\omega^{2} \mathbf{U}_{B}^{j+1}\right)+\sigma_{B} \mathbf{U}_{B}^{j+1}=-\frac{1}{\lambda}\left(R \mathbf{U}_{T}^{j}-\omega^{2} \mathbf{U}_{T}^{j}\right)+\sigma_{B} \mathbf{U}_{T}^{j} \\
& \frac{1}{\lambda}\left(R \mathbf{U}_{T}^{j+1}-\omega^{2} \mathbf{U}_{T}^{j+1}\right)+\sigma_{T} \mathbf{U}_{T}^{j+1}=-\frac{1}{\lambda}\left(R \mathbf{U}_{B}^{j}-\omega^{2} \mathbf{U}_{B}^{j}\right)+\sigma_{T} \mathbf{U}_{B}^{j} .
\end{aligned}
$$

If $\sigma_{B}$ and $\sigma_{T}$ are such that the matrix $\left(R-\omega^{2} I+\lambda \sigma_{L}\right)$ is invertible for $L=B, T$, we have

$$
\begin{aligned}
\mathbf{U}_{B}^{j+1} & =\left(R-\omega^{2} I+\lambda \sigma_{B}\right)^{-1}\left(-R+\omega^{2} I+\lambda \sigma_{B}\right) \mathbf{U}_{T}^{j} \\
\mathbf{U}_{T}^{j+1} & =\left(R-\omega^{2} I+\lambda \sigma_{T}\right)^{-1}\left(-R+\omega^{2} I+\lambda \sigma_{T}\right) \mathbf{U}_{B}^{j} .
\end{aligned}
$$

Given $\mathbf{U}_{B}^{0}$ and $\mathbf{U}_{T}^{0}$, if we let

$$
M_{B}:=\left(R-\omega^{2} I+\lambda \sigma_{B}\right)^{-1}\left(-R+\omega^{2} I+\lambda \sigma_{B}\right)\left(R-\omega^{2} I+\lambda \sigma_{T}\right)^{-1}\left(-R+\omega^{2} I+\lambda \sigma_{T}\right)
$$

and

$$
M_{T}=\left(R-\omega^{2} I+\lambda \sigma_{T}\right)^{-1}\left(-R+\omega^{2} I+\lambda \sigma_{T}\right)\left(R-\omega^{2} I+\lambda \sigma_{B}\right)^{-1}\left(-R+\omega^{2} I+\lambda \sigma_{B}\right),
$$

we can write

$$
\mathbf{U}_{B}^{2 n}=M_{B}^{j} \mathbf{U}_{B}^{0} \quad \mathbf{U}_{T}^{2 n}=M_{T}^{j} \mathbf{U}_{T}^{0} .
$$

Since the matrices $M_{B}$ and $M_{T}$ share the same spectrum as a function of $k_{1}$ and $k_{2}$, we can define the convergence rate of the algorithm as being the spectral radius of $M_{B}$ (or, equivalently, $M_{T}$ ), namely

$$
\rho\left(k_{1}, k_{2}\right):=\rho\left(M_{T}\right)=\rho\left(M_{B}\right) .
$$

Thus, the sequence $\left(\mathbf{U}_{B}^{j}, \mathbf{U}_{T}^{j}\right)$ converges to zero if and only if $\rho<1$.

Notice that if $\sigma_{B}=\sigma_{T}$ then $M_{B}=M_{T}=N^{2}$ with $N=\left(R-\omega^{2} I+\lambda \sigma\right)^{-1}\left(-R+\omega^{2} I+\lambda \sigma\right)$. Moreover, choosing $\sigma_{B}=\sigma_{T}=\frac{1}{\lambda}\left(R-\omega^{2} I\right)$, the operator $R-\omega^{2} I+\lambda \sigma_{L}=2\left(R-\omega^{2} I\right)$ is 
invertible if and only if $\omega \neq 0$ and $\omega^{2} \neq|k|^{2}:=k_{1}^{2}+k_{2}^{2}$ : in this case $\rho\left(M_{T}\right)=\rho\left(M_{B}\right)=0$, and the algorithm converges in two iterations. Unfortunately, due to the presence of the square root in the symbol, this choice corresponds to non-local operators $S_{L}$ which cannot be used in practice in the real domain where computations take place. In order to overcome this problem we replace $\lambda$ with a constant, in other words, we take $\sigma_{L}=\frac{1}{p_{L}}\left(R-\omega^{2} I\right)$ with $p_{L} \in \mathbb{C}$ independent of $k_{1}$ and $k_{2}$. In this way, for $L=B, T, S_{L}$ is the second order operator

$$
S_{L}=\frac{1}{p_{L}}\left(\overrightarrow{\operatorname{curl}}_{\Gamma} \operatorname{curl}_{\Gamma}-\omega^{2} I\right)
$$

where curl $\Gamma_{\Gamma}$ is the surface scalar curl operator and $\overrightarrow{\operatorname{curl}}_{\Gamma}$ the surface vector curl operator.

With this position, the iterative matrix on the interface is

$$
M_{B}=M_{T}=\frac{-p_{B}+\lambda}{p_{B}+\lambda} \frac{-p_{T}+\lambda}{p_{T}+\lambda} I,
$$

and the convergence rate is in fact a function of $|k|^{2}$, which depends only on the choice of the parameters $p_{B}$ and $p_{T}$. In the remaining part of the section we present two suitable choices for such parameters.

\subsection{Symmetric interface conditions}

The most natural choice is to take $p_{B}=p_{T}=p \in \mathbb{C}$, which amounts to use the same operator in the interface condition on both subdomains: we thus refer to this choice as symmetric interface conditions. With our positions, owing to (8), the sequence $\left(\mathbf{U}_{B}^{j}, \mathbf{U}_{T}^{j}\right)$ converges if and only if

$$
\rho(p,|k|)=\left|\frac{-p+\lambda}{p+\lambda}\right|^{2}<1 .
$$

On one hand, for evanescent modes $\left(|k|^{2}>\omega^{2}\right)$, we have that

$$
\left|\frac{-p+\lambda}{p+\lambda}\right|^{2}=\frac{\left(-\mathcal{R} e p+\sqrt{|k|^{2}-\omega^{2}}\right)^{2}+(\mathcal{I} m p)^{2}}{\left(\mathcal{R} e p+\sqrt{|k|^{2}-\omega^{2}}\right)^{2}+(\mathcal{I} m p)^{2}}=\frac{|p|^{2}+|k|^{2}-\omega^{2}-2 \mathcal{R} e p \sqrt{|k|^{2}-\omega^{2}}}{|p|^{2}+|k|^{2}-\omega^{2}+2 \mathcal{R} e p \sqrt{|k|^{2}-\omega^{2}}}<1
$$

if and only if $\mathcal{R} e p>0$.

On the other hand, for propagative modes $\left(|k|^{2}<\omega^{2}\right)$, we similarly have

$$
\left|\frac{-p+\lambda}{p+\lambda}\right|^{2}=\frac{(\mathcal{R} e p)^{2}+\left(-\mathcal{I} m p+\sqrt{\omega^{2}-|k|^{2}}\right)^{2}}{(\mathcal{R} e p)^{2}+\left(\mathcal{I} m p+\sqrt{\omega^{2}-|k|^{2}}\right)^{2}}=\frac{|p|^{2}+\omega^{2}-|k|^{2}-2 \mathcal{I} m p \sqrt{\omega^{2}-|k|^{2}}}{|p|^{2}+\omega^{2}-|k|^{2}+2 \mathcal{I} m p \sqrt{\omega^{2}-|k|^{2}}}<1
$$

if and only if $\mathcal{I} m p>0$.

Notice, however, that for $|k|^{2}=\omega^{2}$ the convergence rate $\rho(p,|k|)=1$ for all $p \in \mathbb{C}$.

This analysis leads us to consider algorithm (4) with the second order interface conditions

$$
\left\{\begin{aligned}
\operatorname{curl} \mathbf{u}_{B}^{j+1} \times \mathbf{n}_{B}+\frac{1}{p}\left(\overrightarrow{\operatorname{curl}}_{\Gamma} \operatorname{curl}_{\Gamma}-\omega^{2} I\right)\left(\mathbf{u}_{B, \tau}^{j+1}\right) & \\
& =\operatorname{curl} \mathbf{u}_{T}^{j} \times \mathbf{n}_{B}+\frac{1}{p}\left(\overrightarrow{\operatorname{curl}}_{\Gamma} \operatorname{curl}{ }_{\Gamma}-\omega^{2} I\right)\left(\mathbf{u}_{T, \tau}^{j}\right) \\
\operatorname{curl} \mathbf{u}_{T}^{j+1} \times \mathbf{n}_{T}+\frac{1}{p}\left(\overrightarrow{\operatorname{curl}}_{\Gamma}\right. & \left.\operatorname{curl}_{\Gamma}-\omega^{2} I\right)\left(\mathbf{u}_{T, \tau}^{j+1}\right) \\
& =\operatorname{curl} \vec{u}_{B}^{j} \times \mathbf{n}_{T}+\frac{1}{p}\left(\overrightarrow{\operatorname{curl}}_{\Gamma} \operatorname{curl}{ }_{\Gamma}-\omega^{2} I\right)\left(\mathbf{u}_{B, \tau}^{j}\right) .
\end{aligned}\right.
$$


on $\Gamma$. The complex parameter $p$ is chosen with $\mathcal{R} e p, \mathcal{I} m p \in \mathbb{R}^{+}$. In the following we call

$$
\mathbb{C}^{+}:=\{q \in \mathbb{C} \mid \mathcal{R} e q>0, \mathcal{I} m q>0\} .
$$

The best choice of $p$ is the one that gives a minimal convergence rate. As we have noted before, if $|k|^{2}=\omega^{2}$ then $\rho(p,|k|)=1$ for all $p \in \mathbb{C}^{+}$. However, following [6], we can restrict ourselves to consider spatial frequencies such that $|k| \in I:=\left(m, \omega_{-}\right) \cup\left(\omega_{+}, M\right)$ where $\omega_{-}$ and $\omega_{+}$are parameters to be chosen such that $\omega_{-}<\omega<\omega_{+}$, where $m$ denotes the smallest value of $|k|$ relevant to the subdomain and where $M$ is the largest value of $|k|$ supported by the numerical grid. This largest frequency is of order $\pi / h$. Hence we choose $p^{\text {opt }}$ as being the solution of the following optimization problem

$$
\rho_{\mathrm{S}}^{\mathrm{opt}}=\min _{p \in \mathbb{C}^{+}} \max _{|k| \in I}|\rho(p,|k|)|
$$

that coincides with the one analyzed in [6], whose solution is given by

$$
\mathcal{R} e p^{\mathrm{opt}}=\mathcal{I} m p^{\mathrm{opt}}=\frac{\left[\left(\omega^{2}-\omega_{-}^{2}\right)\left(M^{2}-\omega^{2}\right)\right]^{1 / 4}}{\sqrt{2}},
$$

and provides the convergence rate

$$
\rho_{\mathrm{S}}^{\mathrm{opt}}=\frac{1-\sqrt{2}\left(\frac{\omega^{2}-\omega_{-}^{2}}{M^{2}-\omega^{2}}\right)^{1 / 4}+\left(\frac{\omega^{2}-\omega_{-}^{2}}{M^{2}-\omega^{2}}\right)^{1 / 2}}{1+\sqrt{2}\left(\frac{\omega^{2}-\omega_{-}^{2}}{M^{2}-\omega^{2}}\right)^{1 / 4}+\left(\frac{\omega^{2}-\omega_{-}^{2}}{M^{2}-\omega^{2}}\right)^{1 / 2}} .
$$

\subsection{Unsymmetric interface conditions}

Another opportunity stems from the expression of matrices $M_{B}$ and $M_{T}$ in (8), and from the fact that $\lambda$ is either real and positive or purely imaginary: choosing one parameter real and positive and the other one purely imaginary (with positive imaginary part), the reduction factor is strictly smaller than 1 for all modes. To fix the ideas, if we take, for instance, $p_{B}=r \in \mathbb{R}^{+}$, and $p_{T}=i q, q \in \mathbb{R}^{+}$, the reduction factor is given by

$$
\rho(r, q,|k|)=\left|\frac{-r+\lambda}{r+\lambda} \frac{-i q+\lambda}{i q+\lambda}\right|
$$

(and we claim that the inverse choice provides the same result). We immediately have, for propagative modes

$$
\left|\frac{-r+\lambda}{r+\lambda}\right|=1 \quad\left|\frac{-i q+\lambda}{i q+\lambda}\right|<1,
$$

as well as, for evanescent modes

$$
\left|\frac{-r+\lambda}{r+\lambda}\right|<1 \quad\left|\frac{-i q+\lambda}{i q+\lambda}\right|=1 .
$$

The algorithm we are thus led to consider is based on the following interface conditions on $\Gamma$ (recall that $(i q)^{-1}=-i / q$ )

$$
\left\{\begin{aligned}
& \operatorname{curl} \mathbf{u}_{B}^{j+1} \times \mathbf{n}_{B}+\frac{1}{r}\left(\overrightarrow{\operatorname{curl}}_{\Gamma}\right.\left.\operatorname{curl}{ }_{\Gamma}-\omega^{2} I\right)\left(\mathbf{u}_{B, \tau}^{j+1}\right) \\
&=\operatorname{curl} \mathbf{u}_{T}^{j} \times \mathbf{n}_{B}+\frac{1}{r}\left(\overrightarrow{\operatorname{curl}}_{\Gamma} \operatorname{curl}_{\Gamma}-\omega^{2} I\right)\left(\mathbf{u}_{T, \tau}^{j}\right) \\
& \operatorname{curl} \mathbf{u}_{T}^{j+1} \times \mathbf{n}_{T}-\frac{i}{q}\left(\overrightarrow{\operatorname{curl}}_{\Gamma} \operatorname{curl}_{\Gamma}-\omega^{2} I\right)\left(\mathbf{u}_{T, \tau}^{j+1}\right) \\
&=\operatorname{curl} \mathbf{u}_{B}^{j} \times \mathbf{n}_{T}-\frac{i}{q}\left(\overrightarrow{\operatorname{curl}}_{\Gamma} \operatorname{curl}{ }_{\Gamma}-\omega^{2} I\right)\left(\mathbf{u}_{B, \tau}^{j}\right) .
\end{aligned}\right.
$$


As this choice entails the use of different operators in the construction of matching conditions on the two sides of the interface, we refer to it as unsymmetric interface conditions.

Again, the best choice of the parameters $p_{B}=r$ and $p_{T}=i q$ is the one that gives a minimal convergence rate. Once again, if $|k|^{2}=\omega^{2}$ then $\rho(r, q,|k|)=1$ for each $r, q \in \mathbb{R}$. However, in this case, owing to (12) and (13), an optimization procedure for the parameters can be performed separately on the two intervals $I_{1}=\left(m, \omega_{-}\right)$and $I_{2}=\left(\omega_{+}, M\right)$, where $m, M, \omega_{-}$ and $\omega_{+}$are the ones introduced in the previous section. The min-max problem we are dealing with is thus decoupled and reads

$$
\rho_{\mathrm{NS}}^{\mathrm{opt}}= \begin{cases}\min _{r \in \mathbb{R}^{+}} \max _{|k| \in I_{1}}\left|\frac{-r+\lambda}{r+\lambda}\right| & \text { for propagative modes } \\ \min _{q \in \mathbb{R}^{+}} \max _{|k| \in I_{2}}\left|\frac{-i q+\lambda}{i q+\lambda}\right| & \text { for evanescent modes }\end{cases}
$$

which has an elegant analytical solution, given by the following lemma.

Lemma 2 The solution of the minimization problem (15) is given by

$$
\begin{cases}r^{\mathrm{opt}}=\left[\left(\omega^{2}-\omega_{-}^{2}\right)\left(\omega^{2}-m^{2}\right)\right]^{1 / 4} & \text { for propagative modes } \\ q^{\mathrm{opt}}=\left[\left(\omega_{+}^{2}-\omega^{2}\right)\left(M^{2}-\omega^{2}\right)\right]^{1 / 4} & \text { for evanescent modes }\end{cases}
$$

and the optimal reduction factor of the algorithm is given by

$$
\rho_{\mathrm{NS}}^{\mathrm{opt}}= \begin{cases}\frac{\left(\omega^{2}-\omega_{-}^{2}\right)^{1 / 4}-\left(\omega^{2}-m^{2}\right)^{1 / 4}}{\left(\omega^{2}-\omega_{-}^{2}\right)^{1 / 4}+\left(\omega^{2}-m^{2}\right)^{1 / 4}} & \text { for propagative modes } \\ \frac{\left(M^{2}-\omega^{2}\right)^{1 / 4}-\left(\omega_{+}^{2}-\omega^{2}\right)^{1 / 4}}{\left(M^{2}-\omega^{2}\right)^{1 / 4}+\left(\omega_{+}^{2}-\omega^{2}\right)^{1 / 4}} & \text { for evanescent modes }\end{cases}
$$

Proof. We outline the solution for propagative modes, the solution for evanescent ones being similar. With the change of variables

$$
x:=\sqrt{\omega^{2}-|k|^{2}}
$$

we are led to study

$$
\min _{r \in \mathbb{R}^{+}} \max _{x \in I^{-}}\left|\frac{x-r}{x+r}\right|,
$$

where $I^{-}=\left(\sqrt{\omega^{2}-\omega_{-}^{2}}, \sqrt{\omega^{2}-m^{2}}\right)=\left(x_{0}, x_{1}\right)$. Since the function $\varphi(x, r)=\frac{x-r}{x+r}$ is monotone increasing for $x \in I^{-}, \varphi(0, r)=-1$, and $\lim _{x \rightarrow \infty} \varphi(x, r)=1$, the maximum of the modulus is attained on the boundary of the interval. The min-max problem reduces thus to

$$
\min _{r \in \mathbb{R}^{+}} \max \left\{\left|\frac{x_{0}-r}{x_{0}+r}\right|,\left|\frac{x_{1}-r}{x_{1}+r}\right|\right\}
$$


We thus have that the optimal parameter for propagative modes belongs to the interval $\left(x_{0}, x_{1}\right)$, and is given by

$$
r^{\mathrm{opt}}=\sqrt{x_{0} x_{1}}=\left[\left(\omega^{2}-\omega_{-}^{2}\right)\left(\omega^{2}-m^{2}\right)\right]^{1 / 4} .
$$

For evanescent modes, in a similar way, the change of variable $x:=\sqrt{|k|^{2}-\omega^{2}}$, reduces to study the min-max problem on $\mathbb{R}^{+} \times I^{+}$, where $I^{+}=\left(\sqrt{\omega_{+}^{2}-\omega^{2}}, \sqrt{M^{2}-\omega^{2}}\right)$, whose solution is given by

$$
q^{\mathrm{opt}}=\left[\left(\omega_{+}^{2}-\omega^{2}\right)\left(M^{2}-\omega^{2}\right)\right]^{1 / 4}
$$

So far, a simple algebra leads to the expression of the optimal reduction factor (16).

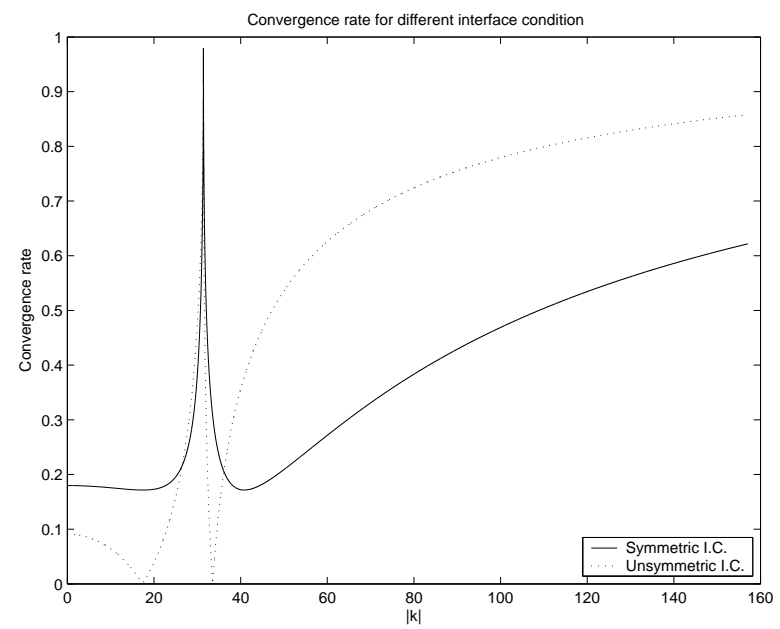

Figure 1: Convergence rate (as a function of $|k|$ ) in Fourier space of the optimized Schwarz algorithm with symmetric (solid line) and unsymmetric (dashed line) interface conditions.

We report in Figure 1 the convergence rates $\rho\left(p^{\mathrm{opt}},|k|\right)$ and $\rho\left(r^{\mathrm{opt}}, q^{\mathrm{opt}},|k|\right)$.

\section{Two domain decomposition}

In the following we consider a bounded domain $\Omega \subset \mathbb{R}^{3}$ and the boundary value problem

$$
\begin{cases}\operatorname{curl} \operatorname{curl} \mathbf{u}-\omega^{2} \mathbf{u}=\mathbf{F} & \text { in } \Omega \\ \operatorname{curl} \mathbf{u} \times \mathbf{n}+i \omega \mathbf{u}=\mathbf{0} & \text { on } \Gamma_{R} \\ \mathbf{u} \times \mathbf{n}=\mathbf{0} & \text { on } \Gamma_{D}\end{cases}
$$

where $\Gamma_{R}$ and $\Gamma_{D}$ are two disjoint open subsets of $\partial \Omega$ such that $\overline{\Gamma_{R}} \cup \overline{\Gamma_{D}}=\partial \Omega$.

We decompose the domain $\Omega$ in two non overlapping subdomains $\Omega_{l}, l=1,2$. We set $\Gamma=\overline{\Omega_{1}} \cap \overline{\Omega_{2}}$ and for simplicity we assume that $\bar{\Gamma} \cap \overline{\Gamma_{R}}=\emptyset$. Let us set $\Gamma_{R, l}=\Gamma_{R} \cap \partial \Omega_{l}$ and $\Gamma_{D, l}=\Gamma_{D} \cap \partial \Omega_{l}$. We denote $\mathbf{n}_{l}$ the unit normal outward vector on $\partial \Omega_{l} \cap \Gamma$. 
We consider the following iterative procedure:

$$
\begin{aligned}
& \begin{cases}\operatorname{curl} \operatorname{curl}_{\mathbf{u}_{1}^{j+1}-\omega^{2} \mathbf{u}_{1}^{j+1}=\mathbf{F}} & \text { in } \Omega_{1} \\
\operatorname{curl} \mathbf{u}_{1}^{j+1} \times \mathbf{n}_{1}+i \omega \mathbf{u}_{1, \tau}^{j+1}=\mathbf{0} & \text { on } \Gamma_{R, 1} \\
\mathbf{u}_{1}^{j+1} \times \mathbf{n}_{1}=\mathbf{0} & \text { on } \Gamma_{D, 1} \\
\operatorname{curl} \mathbf{u}_{1}^{j+1} \times \mathbf{n}_{1}+\frac{1}{p_{1}}\left(\overrightarrow{\operatorname{curl}}_{\Gamma} \operatorname{curl}_{\Gamma}-\omega^{2} I\right)\left(\mathbf{u}_{1, \tau}^{j+1}\right) & \\
\quad=\operatorname{curl} \mathbf{u}_{2}^{j} \times \mathbf{n}_{1}+\frac{1}{p_{1}}\left(\overrightarrow{\operatorname{curl}}_{\Gamma} \operatorname{curl}_{\Gamma}-\omega^{2} I\right)\left(\mathbf{u}_{2, \tau}^{j}\right) & \text { on } \Gamma ;\end{cases}
\end{aligned}
$$

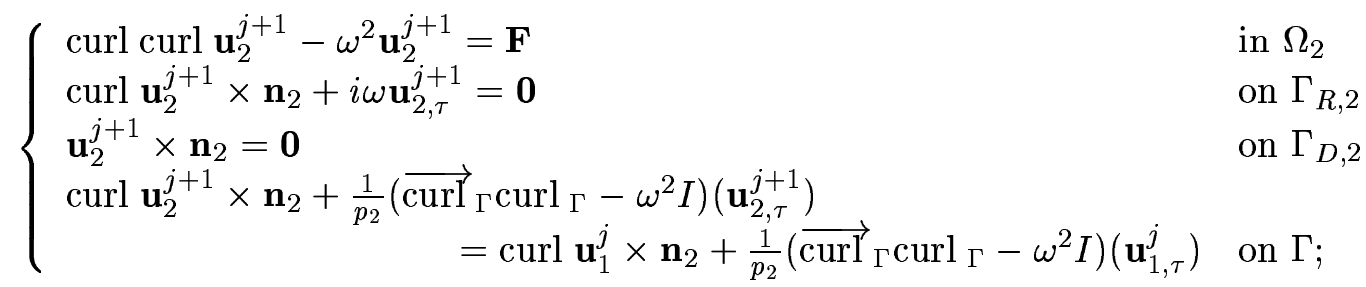

where either $p_{1}=p_{2}=p^{\mathrm{opt}}$, or $p_{1}=r^{\mathrm{opt}}$, and $p_{2}=i q^{\mathrm{opt}}$, according to the choice of symmetric or unsymmetric interface conditions. By introducing the new variables

$$
\lambda_{1}^{j}:=\operatorname{curl} \mathbf{u}_{2}^{j} \times \mathbf{n}_{1}+\frac{1}{p_{1}}\left(\overrightarrow{\operatorname{curl}}_{\Gamma} \operatorname{curl}{ }_{\Gamma}-\omega^{2} I\right)\left(\mathbf{u}_{2, \tau}^{j}\right)
$$

and

$$
\lambda_{2}^{j}:=\operatorname{curl} \mathbf{u}_{1}^{j} \times \mathbf{n}_{2}+\frac{1}{p_{2}}\left(\overrightarrow{\operatorname{curl}}_{\Gamma} \operatorname{curl}{ }_{\Gamma}-\omega^{2} I\right)\left(\mathbf{u}_{1, \tau}^{j}\right)
$$

the algorithm becomes

$$
\begin{aligned}
& \begin{cases}\operatorname{curl} \operatorname{curl} \mathbf{u}_{1}^{j+1}-\omega^{2} \mathbf{u}_{1}^{j+1}=\mathbf{F} & \text { in } \Omega_{1} \\
\operatorname{curl} \mathbf{u}_{1}^{j+1} \times \mathbf{n}_{1}+i \omega \mathbf{u}_{1, \tau}^{j+1}=\mathbf{0} & \text { on } \Gamma_{R, 1} \\
\mathbf{u}_{1}^{j+1} \times \mathbf{n}_{1}=\mathbf{0} & \text { on } \Gamma_{D, 1} \\
\operatorname{curl} \mathbf{u}_{1}^{j+1} \times \mathbf{n}_{1}+\frac{1}{p_{1}}\left(\overrightarrow{\operatorname{curl}}_{\Gamma} \operatorname{curl}_{\Gamma}-\omega^{2} I\right)\left(\mathbf{u}_{1, \tau}^{j+1}\right)=\lambda_{1}^{j} & \text { on } \Gamma ;\end{cases} \\
& \begin{cases}\operatorname{curl} \operatorname{curl}_{\mathbf{u}_{2}^{j+1}-\omega^{2} \mathbf{u}_{2}^{j+1}=\mathbf{F}} & \text { in } \Omega_{2} \\
\operatorname{curl} \mathbf{u}_{2}^{j+1} \times \mathbf{n}_{2}+i \omega \mathbf{u}_{2, \tau}^{j+1}=\mathbf{0} & \text { on } \Gamma_{R, 2} \\
\mathbf{u}_{2}^{j+1} \times \mathbf{n}_{2}=\mathbf{0} & \text { on } \Gamma_{D, 2} \\
\operatorname{curl} \mathbf{u}_{2}^{j+1} \times \mathbf{n}_{2}+\frac{1}{p_{2}}\left(\overrightarrow{\operatorname{curl}}_{\Gamma} \operatorname{curl}{ }_{\Gamma}-\omega^{2} I\right)\left(\mathbf{u}_{2, \tau}^{j+1}\right)=\lambda_{2}^{j} & \text { on } \Gamma ;\end{cases} \\
& \lambda_{1}^{j+1}=-\lambda_{2}^{j}+\left(\frac{1}{p_{1}}+\frac{1}{p_{2}}\right)\left(\overrightarrow{\operatorname{curl}}_{\Gamma} \operatorname{curl}{ }_{\Gamma}-\omega^{2} I\right)\left(\mathbf{u}_{2, \tau}^{j+1}\right) \\
& \lambda_{2}^{j+1}=-\lambda_{1}^{j}+\left(\frac{1}{p_{1}}+\frac{1}{p_{2}}\right)\left(\overrightarrow{\operatorname{curl}}_{\Gamma} \operatorname{curl} \Gamma_{\Gamma}-\omega^{2} I\right)\left(\mathbf{u}_{1, \tau}^{j+1}\right) .
\end{aligned}
$$

\subsection{Finite elements discretization}

We will employ a curl-conforming finite element approximation of the problem. We can use the first family of Nédélec finite elements in a tetrahedral mesh or in a hexaedral mesh (see Nédélec [9]). Other possibles choices are the finite element families introduced and analysed by Nédélec in [10]. 
Let $\left\{\mathcal{T}_{h}\right\}_{h>0}$ be a regular family of finite element meshes of $\Omega$ of elements of maximum diameter $h$ such that each $K \in \mathcal{T}_{h}$ is contained either in $\overline{\Omega_{1}}$ or in $\overline{\Omega_{2}}$. We denote $\mathcal{T}_{l, h}$ the restriction of $\mathcal{T}_{h}$ to $\Omega_{l}, l=1,2$.

Let $\mathbf{P}_{k}, k \geq 1$, be the space of polynomials of degree less than or equal to $k$, and denote by $\mathbf{P}_{k}^{*}$ the space of homogeneous polynomials of degree $k$. We set

$$
\Sigma_{k}:=\left\{\mathbf{p} \in\left(\mathbf{P}_{k}^{*}\right)^{3} \mid \mathbf{p}(\mathbf{x}) \cdot \mathbf{x}=0\right\} \quad, \quad R_{k}:=\left(\mathbf{P}_{k-1}\right)^{3} \oplus \Sigma_{k} .
$$

Notice that $\left(\mathbf{P}_{k-1}\right)^{3} \subset R_{k} \subset\left(\mathbf{P}_{k}\right)^{3}$. In order to discuss also finite elements built on hexaedra, we also need the polynomial space

$Q_{n_{1}, n_{2}, n_{3}}:=$ ppolyomials of maximum degree $n_{1}$ in $x_{1}, n_{2}$ in $x_{2}$, and $n_{3}$ in $\left.x_{3}\right\}$.

If $\left\{\mathcal{T}_{h}\right\}_{h>0}$ is a family of tetrahedral meshes, we employ the finite element spaces

$$
N_{l, h}^{k}:=\left\{\mathbf{v}_{h} \in H\left(\operatorname{curl} ; \Omega_{l}\right) \mid \mathbf{v}_{h \mid K} \in R_{k} \forall K \in \mathcal{T}_{l, h}\right\}
$$

On the other hand, if $\left\{\mathcal{T}_{h}\right\}_{h>0}$ is a family of hexaedral meshes, then the curl-conforming finite element space that we employ is given by

$$
N_{l, h}^{k}:=\left\{\mathbf{v}_{h} \in H\left(\operatorname{curl} ; \Omega_{l}\right) \mid \mathbf{v}_{h \mid K} \in Q_{k-1, k, k} \times Q_{k, k-1, k} \times Q_{k, k, k-1} \forall K \in \mathcal{T}_{l, h}\right\} .
$$

Now we consider the spaces

$$
X_{l, h}=\left\{\mathbf{v}_{h} \in N_{l, h}^{k} \mid \mathbf{v}_{h} \times \mathbf{n}_{l}=\mathbf{0} \text { in } \Gamma_{D, l}\right\} .
$$

A mesh $\mathcal{T}_{l, h}$ of $\Omega_{l}$ induces a mesh on $\Gamma$ in the sense that the faces of the elements in $\mathcal{T}_{l, h}$ that lie on $\Gamma$ obey the usual finite elements meshing constraints. Since we have matching grids in the interface, $\mathcal{T}_{1, h}$ and $\mathcal{T}_{2, h}$ induce the same partition of $\Gamma$. Moreover the tangential component of an element of $N_{l, h}^{k}$ on $\Gamma$ lies in the appropriate two-dimensional analogue of the set $N_{l, h}^{k}$ (that is just the rotated of the Raviart-Thomas finite elements space). Let us denote $Y_{h}$ the finite elements space induced on $\Gamma$ by the tangential components of functions of $N_{l, h}$.

Let $\left\{\mathbf{w}_{i}^{l}\right\}_{i=1}^{N_{l}}$ be a basis of $X_{l, h}$ and $\left\{\mathbf{q}_{i}\right\}_{i=1}^{n}$ the corresponding basis of $Y_{h}$. For simplicity we assume that these basis are such that $\mathbf{q}_{i}=\mathbf{w}_{N_{l}-n+i, \tau}$ for $i=1, \ldots, n, l=1,2$. We consider the matrices $A_{l}, l=1,2$ and $S$ with entries

$$
\begin{gathered}
\left(A_{l}\right)_{i, j}=\int_{\Omega_{l}}\left(\operatorname{curl} \mathbf{w}_{j}^{l} \cdot \operatorname{curl} \mathbf{w}_{i}^{l}-\omega^{2} \mathbf{w}_{j}^{l} \cdot \mathbf{w}_{i}^{l}\right)+i \omega \int_{\Gamma_{R, l}} \mathbf{w}_{j, \tau}^{l} \cdot \mathbf{w}_{i, \tau}^{l} \quad 1 \leq i, j \leq N_{l} \\
S_{i, j}=\int_{\Gamma}\left(\operatorname{curl}_{\Gamma} \mathbf{q}_{j} \operatorname{curl}_{\Gamma} \mathbf{q}_{i}-\omega^{2} \mathbf{q}_{j} \cdot \mathbf{q}_{i}\right) \quad 1 \leq i, j \leq n .
\end{gathered}
$$

Moreover we conside the matrices

$$
B_{l} \in \mathbb{R}^{n \times N_{l}} \quad B_{l}=\left[\begin{array}{ll}
0 & I
\end{array}\right]
$$

where 0 denotes the $n \times\left(N_{l}-n\right)$ matrix with all zero entries, and $I$ is the $n \times n$ identity matrix. $B_{1}$ and $B_{2}$ are the trace operators of domains $\Omega_{1}$ and $\Omega_{2}$ on the interface $\Gamma$. Finally let us consider the vectors

$$
\left(\mathbf{f}_{l}\right)_{j}=\int_{\Omega_{l}} \mathbf{F} \cdot \mathbf{w}_{j}^{l} \quad 1 \leq j \leq N_{l} .
$$

Then, the discrete analogous of algorithm (20) reads: 
Algorithm 1 Given $\Lambda_{l}^{0} \in \mathbb{C}^{n}$, for $j \geq 0$ solve

$$
\left(A_{l}+\frac{1}{p_{l}} B_{l}^{T} S_{l}\right) U_{l}^{j+1}=\mathbf{f}_{L}+B_{l}^{T} \Lambda_{l}^{j}
$$

for $l=1,2$ and then set

$$
\begin{aligned}
& \Lambda_{1}^{j+1}=-\Lambda_{2}^{j}+\left(\frac{1}{p_{1}}+\frac{1}{p_{2}}\right) S B_{2} U_{2}^{j+1}, \\
& \Lambda_{2}^{j+1}=-\Lambda_{1}^{j}+\left(\frac{1}{p_{1}}+\frac{1}{p_{2}}\right) S B_{1} U_{1}^{j+1} .
\end{aligned}
$$

Denoting $R_{l}=A_{l}+\frac{1}{p_{l}} B_{l}^{T} S_{l}$ and $\mathbf{g}_{l}=R_{l}^{-1} \mathbf{f}_{l}$ this algorithm can be rewritten as

$$
\begin{aligned}
& \Lambda_{1}^{j+1}=-\Lambda_{2}^{j}+\left(\frac{1}{p_{1}}+\frac{1}{p_{2}}\right) S B_{2}\left(\mathrm{~g}_{2}+R_{2}^{-1} B_{2}^{T} \Lambda_{2}^{j}\right) \\
& \Lambda_{2}^{j+1}=-\Lambda_{1}^{j}+\left(\frac{1}{p_{1}}+\frac{1}{p_{2}}\right) S B_{1}\left(\mathbf{g}_{1}+R_{1}^{-1} B_{1}^{T} \Lambda_{1}^{j}\right),
\end{aligned}
$$

which is a fixed point iteration to solve the linear system

$$
\left[\begin{array}{cc}
I & I-\left(\frac{1}{p_{1}}+\frac{1}{p_{2}}\right) S B_{2}^{T} R_{2}^{-1} B_{2} \\
I-\left(\frac{1}{p_{1}}+\frac{1}{p_{2}}\right) S B_{1}^{T} R_{1}^{-1} B_{1} & I
\end{array}\right]\left[\begin{array}{c}
\Lambda_{1} \\
\Lambda_{2}
\end{array}\right]=\left(\frac{1}{p_{1}}+\frac{1}{p_{2}}\right)\left[\begin{array}{c}
S B_{1} \mathbf{g}_{1} \\
S B_{2} \mathbf{g}_{2}
\end{array}\right]
$$

As done in [6], instead of solving the fixed point iteration (21) one can use a Krylov subspace method directly on the substructured problem (22). This corresponds to use the optimized Schwarz method as a preconditioner for the Krylov subspace method.

Algorithm 2 Solve (22) using GMRES.

Notice that when the GMRES method is applied to the linear system (22), each multiplication of the matrix

$$
M:=\left[\begin{array}{cc}
I & I-\left(\frac{1}{p_{1}}+\frac{1}{p_{2}}\right) S B_{2}^{T} R_{2}^{-1} B_{2} \\
I-\left(\frac{1}{p_{1}}+\frac{1}{p_{2}}\right) S B_{1}^{T} R_{1}^{-1} B_{1} & I
\end{array}\right]
$$

by a vector requires the solution of two local problems of the type $R_{l} \mathbf{x}=\mathbf{b}, l=1,2$.

\section{Numerical Experiments}

In this section we validate the interface conditions proposed in the previous sections by means of some numerical tests. For the implementation we have used MATLAB ${ }^{T M}$ 6.5. We compare the performance of the two type of interface conditions for both algorithm 1 and 2 . To solve the non hermitian linear systems of the form $R_{l} \mathbf{x}=\mathbf{b}, l=1,2$, we use the COCG algorithm proposed in [13].

The computational domain throughout this section will be

$$
\Omega=[0,2] \times[0,1] \times[0,1],
$$


in which we consider the following problem:

$$
\begin{cases}\operatorname{curl} \operatorname{curl} \mathbf{u}-\omega^{2} \mathbf{u}=\mathbf{F} & \text { in }[0,2] \times[0,1] \times[0,1] \\ \mathbf{u} \times \mathbf{n}=\mathbf{0} & \text { on }\{y=0\},\{y=1\},\{z=0\},\{z=1\} \\ \operatorname{curl} \mathbf{u} \times \mathbf{n}+i \omega \mathbf{u}_{\tau}=\mathbf{0} & \text { on }\{x=0\},\{x=2\} .\end{cases}
$$

We take $\omega=5 \pi$. The function $\mathbf{F}$ is chosen such that the exact solution $\mathbf{u}$ is the gradient of the function $\Psi=x(2-x) y(1-y) z(1-z)$.

We employ curl-conforming linear finite elements in a uniform hexaedral mesh and we perform the computations with the optimized parameters $p^{\text {opt }}, r^{\text {opt}}$, and $q^{\text {opt }}$ as defined in the previous sections.

\subsection{Influence of the mesh size}

In this first series of tests we decompose $\Omega$ into two equal subdomains, $\Omega_{1}=[0,1] \times[0,1] \times[0,1]$, and $\Omega_{2}=[1,2] \times[0,1] \times[0,1]$.

In Table 1 we report the number of iteration required to achive a residual vector of norm less than $10^{-7}$. We compare the four algorithms for different meshsizes. In Figure 2 we

\begin{tabular}{|c||c|c|c|c|}
\hline$h$ & Iterative S & Iterative NS & Krylov S & Krylov NS \\
\hline $1 / 8$ & 35 & 36 & 9 & 14 \\
\hline $1 / 12$ & 36 & 41 & 11 & 17 \\
\hline $1 / 16$ & 55 & 63 & 12 & 19 \\
\hline $1 / 20$ & 77 & 111 & 12 & 21 \\
\hline
\end{tabular}

Table 1: Iteration counts for different algorithms and different meshsizes

report the convergence histories of the four algorithms for a fixed mesh size, and in Figure 3 we report the convergence histories of each algorithm with respect to the mesh size.

The most efficient method is the Krylov one with symmetric interface condition. The speed-up of the Krylov accelerations is remarkable for both symmetric and unsymmetric interface conditions (no wonder in that). Evidence is that the symmetric interface condition (i.e. the use of the same operator on both sides of the interface) provides a faster convergence, which becomes more evident for small values of $h$ (see Figure 2).

\subsection{Influence of the position of the interface}

In the previous tests the two subdomains where symmetric with respect to the interface. In the second series of tests we compare the performance of the four algorithms when the position of the interface changes. We choose $h=1 / 12$, and we consider two different positions of the interface, at $x=1 / 3$, and $x=2 / 3$, yielding the following decompositions:

$$
\Omega_{1}=[0,1 / 3] \times[0,1]^{2}, \quad \Omega_{2}=[1 / 3,2] \times[0,1]^{2}
$$

and

$$
\Omega_{1}=[0,2 / 3] \times[0,1]^{2}, \quad \Omega_{2}=[2 / 3,2] \times[0,1]^{2} .
$$



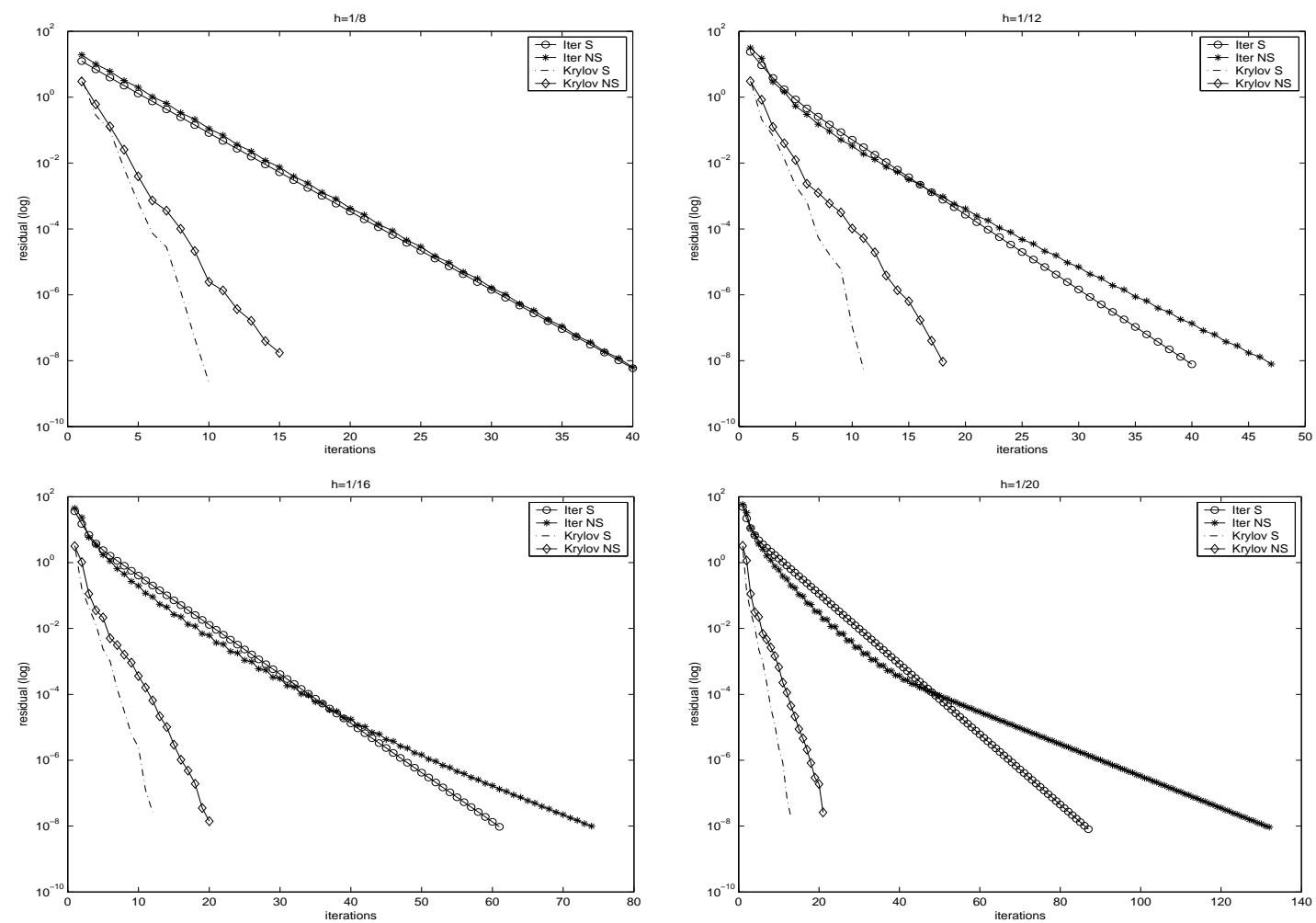

Figure 2: Convergence history of the different algorithms for different mesh sizes: from top left $h=1 / 8, h=1 / 12, h=1 / 16, h=1 / 20$.

\begin{tabular}{|c||c|c|c|c|}
\hline$\Omega_{1}$ & Iterative S & Iterative NS & Krylov S & Krylov NS \\
\hline$[0,1 / 3] \times[0,1]^{2}$ & 37 & 43 & 15 & 17 \\
\hline$[0,2 / 3] \times[0,1]^{2}$ & 36 & 41 & 14 & 17 \\
\hline$[0,1] \times[0,1]^{2}$ & 36 & 41 & 11 & 17 \\
\hline
\end{tabular}

Table 2: Iteration counts for different position of the interface, $h=1 / 12$

We report in Table 2 the iteration counts for the four algorithms, which appear to be quite insensitive to the position of the interface, and, consequentely, to the size of the subdomains. We stop the iterations when the residual error has norm less that $10^{-7}$. Only the Krylov algorithm with symmetric interface conditions shows a certain increase in the number of iteration, which is in any case low: this algorithm remains in fact the fastest one.

\section{Other interface conditions used in DDM methods for the harmonic Maxwell's system}

In this section we give a brief survey of other interface conditions proposed in literature for the harmonic Maxwell system, always in the framework of non-overlapping domain decomposition methods. We apply to these interface conditions the analysis we made in Section 2 and we 

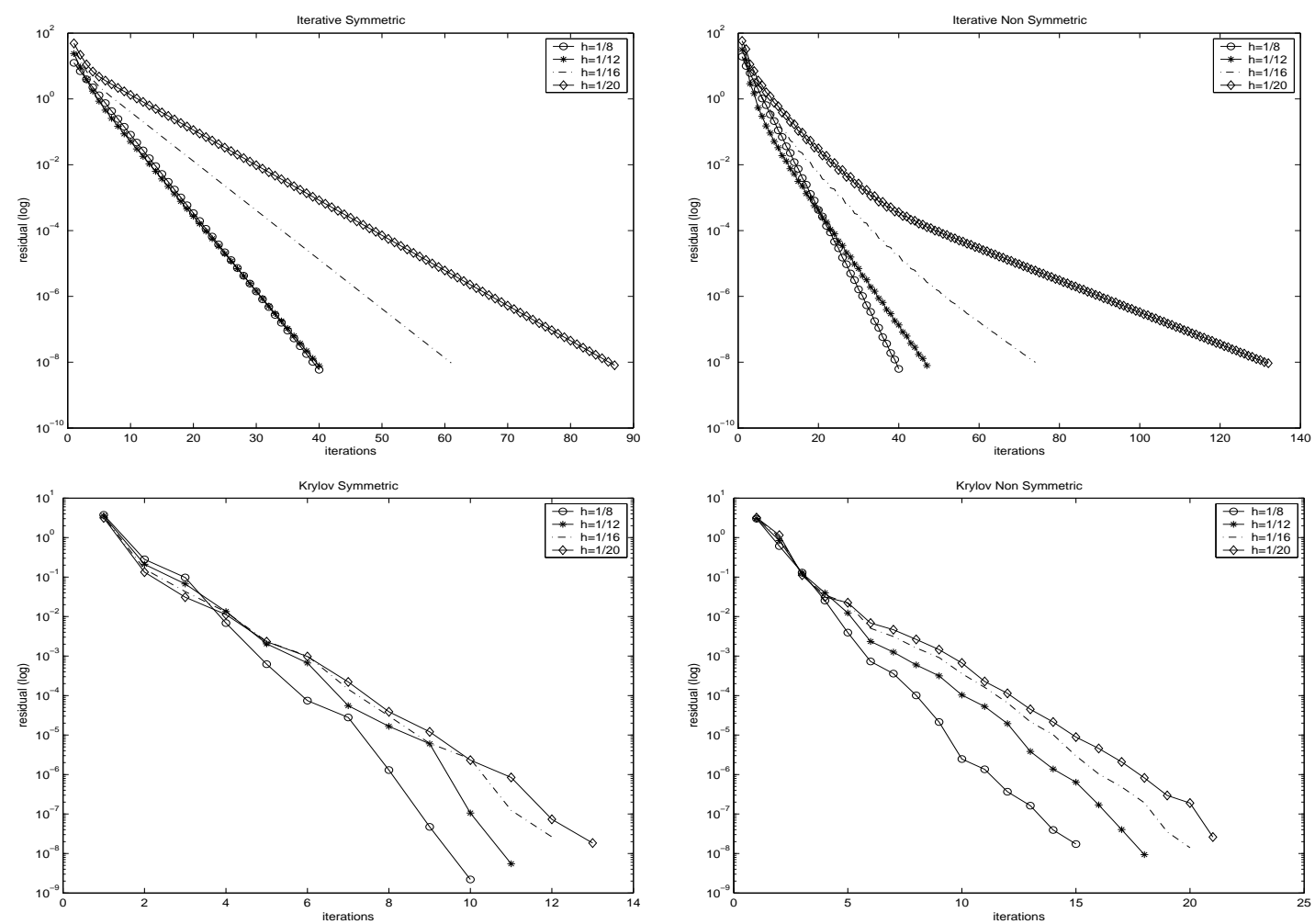

Figure 3: Convergence history of the different algorithms with respect to the mesh size: from top left Iterative Symmetric, Iterative Non-Symmetric, Krylov Symmetric, Krylov NonSymmetric

note that for all of them the iterative procedure (6) fails to converge, in the presence of evanescent modes. We will keep throughout this section the notations used in Section 2.

\section{B. Despres - P. Joly - J.E. Roberts [5]}

The earliest non-overlapping domain decomposition algorithm for Maxwell system has been proposed in [5], where the interface operator is given by

$$
S_{B}=S_{T}=i \omega I .
$$

In [5], the authors, considering a decomposition into an arbitrary number of subdomains and a boundary condition of radiative type at finite distance, show that the proposed algorithm converges weakly in $H$ (curl,$\left.\Omega_{j}\right),(j=1, . ., N)$ to the solution of the single domain problem, and that $\mathbf{u}_{j}^{n}$ and curl $\mathbf{u}_{j}^{n} \times \mathbf{n}$ converge to $\mathbf{u}_{j}$ and curl $\mathbf{u}_{j} \times \mathbf{n}$ respectively. The convergence properties of this algorithm depend heavily on the boundary condition, as it allows to control the energy on the outer boundaries by means of the energy on the inner ones.

For the interface operator (24) the Fourier analysis shows that the iterative procedure (6) converges only for propagative modes, while for the evanescent ones the convergence rate is exactly 1 . 
In fact, in this case we have $M_{B}=M_{T}=N^{2}$, where

$$
N=\frac{1}{(\lambda+i \omega)^{2}}\left[\begin{array}{cc}
k_{1}^{2}-k_{2}^{2} & 2 k_{1} k_{2} \\
2 k_{1} k_{2} & k_{2}^{2}-k_{1}^{2}
\end{array}\right]
$$

and the eigenvalues of $N$ are

$$
\mu^{ \pm}= \pm \frac{|k|^{2}}{(\lambda+i \omega)^{2}}
$$

If $|k|^{2}<\omega^{2}$ then $\lambda=i \sqrt{\omega^{2}-|k|^{2}}$ and

$$
\left|\mu^{ \pm}\right|=\frac{|k|^{2}}{\left(\sqrt{\omega^{2}-|k|^{2}}+\omega\right)^{2}}<\frac{\omega^{2}}{\left(\sqrt{\omega^{2}-|k|^{2}}+\omega\right)^{2}}<1 .
$$

On the other hand if $|k|^{2}>\omega^{2}$ then $\lambda$ is real and

$$
\left|\mu^{ \pm}\right|=\frac{|k|^{2}}{\lambda^{2}+\omega^{2}}=1 .
$$

Thus, the algorithm (4) with the interface conditions proposed in [5] does not converge for evanescent modes. However, the boundary condition (a radiation at finite distance) considered by the authors does not allow evenescent modes to come into the system: thus, the above result is not in contradiction with their work.

\section{P. Chevalier [2]}

To improve the convergence of the algorithm introduced in [5], $\mathrm{P}$. Chevalier proposed in his thesis [2] the use of the interface operator

$$
S_{B}=S_{T}=i \omega I+\zeta \overrightarrow{\operatorname{curl}}_{\Gamma} \operatorname{curl} \Gamma
$$

$\zeta \in \mathbb{C}$ being a complex parameter to be tuned, which consists in adding to Després' interface conditions a space derivative of second order in the direction tangential to the interface, following what is done in literature for Helmholtz equations (see for instance [2] and [6]).

In [2] there is neither an accurate analysis of the algorithm, nor a clear tuning of the parameter $\zeta$, and we show here that, differently from the case of Helmholtz equation, the use of the second order transmission condition does not guarantee convergence for evanescent modes.

In fact, we have again $M_{B}=M_{T}=N^{2}$, with

$$
\begin{aligned}
N & =\left[R-\omega^{2} I+\lambda(\zeta R+i \omega I)\right]^{-1}\left[-R+\omega^{2} I+\lambda(\zeta R+i \omega I)\right] \\
& =\left[(\lambda \zeta+1) R+\left(i \lambda \omega-\omega^{2}\right) I\right]^{-1}\left[(\lambda \zeta-1) R+\left(i \lambda \omega+\omega^{2}\right) I\right] .
\end{aligned}
$$

If $\lambda \zeta \neq \pm 1$

$$
=\frac{\lambda \zeta-1}{\lambda \zeta+1}\left[R+\frac{i \lambda \omega-\omega^{2}}{\lambda \zeta+1} I\right]^{-1}\left[R+\frac{i \lambda \omega+\omega^{2}}{\lambda \zeta-1} I\right] .
$$


Setting $a=\frac{i \lambda \omega-\omega^{2}}{\lambda \zeta+1}$ and $b=\frac{i \lambda \omega+\omega^{2}}{\lambda \zeta-1}$,

$$
\begin{aligned}
N & =\frac{\lambda \zeta-1}{\lambda \zeta+1}(R+a I)^{-1}(R+b I) \\
& =\frac{\lambda \zeta-1}{\lambda \zeta+1} \frac{1}{a|k|^{2}+a^{2}}\left[\begin{array}{cc}
k_{1}^{2}+a & k_{1} k_{2} \\
k_{1} k_{2} & k_{2}^{2}+a
\end{array}\right]\left[\begin{array}{cc}
k_{2}^{2}+b & -k_{1} k_{2} \\
-k_{1} k_{2} & k_{1}^{2}+b
\end{array}\right] \\
& =\frac{\lambda \zeta-1}{\lambda \zeta+1} \frac{1}{a|k|^{2}+a^{2}}\left[\begin{array}{cc}
k_{1}^{2} b+k_{2}^{2} a+a b & -k_{1} k_{2}(a-b) \\
-k_{1} k_{2}(a-b) & k_{1}^{2} a+k_{2}^{2} b+a b
\end{array}\right]
\end{aligned}
$$

The eigenvalues of the matrix $\left[\begin{array}{cc}k_{1}^{2} b+k_{2}^{2} a+a b & -k_{1} k_{2}(a-b) \\ -k_{1} k_{2}(a-b) & k_{1}^{2} a+k_{2}^{2} b+a b\end{array}\right]$ are $\gamma^{+}=a\left(|k|^{2}+b\right)$ and $\gamma^{-}=b\left(|k|^{2}+a\right)$, hence the eigenvalues of $N$ are

$$
\mu^{+}=\frac{\lambda \zeta-1}{\lambda \zeta+1} \frac{|k|^{2}+b}{|k|^{2}+a}, \quad \mu^{-}=\frac{\lambda \zeta-1}{\lambda \zeta+1} \frac{b}{a}=\frac{i \lambda+\omega}{i \lambda-\omega} .
$$

It is now easy to observe that when $\lambda$ is real $\left|\mu^{-}\right|=1$, and the algorithm does not converge for evanescent modes.

\section{F. Collino - G. Delbue - P. Joly - A. Piacentini [3]}

The Fourier analysis in Section 2 (Lemma 1 and Remark 1) shows that the operator linking $\mathbf{n} \times \mathbf{u} \times \mathbf{n}$ to curl $\mathbf{u} \times \mathbf{n}$ is

$$
\mathcal{T}(\operatorname{curl} \mathbf{u} \times \mathbf{n})+\left(\omega^{2}-\overrightarrow{\operatorname{curl}}_{\Gamma} \operatorname{curl}{ }_{\Gamma}\right) \mathbf{n} \times \mathbf{u} \times \mathbf{n}=0,
$$

where $\mathcal{T}$ is a pseudodifferential operator corresponding, in the Fourier space, to the multiplication by $\lambda(|k|)=\sqrt{|k|^{2}-\omega^{2}}$ : due to its non-locality, this operator cannot be used in practice.

Another interface condition, based on a zero-th order Taylor expansion of the function $\lambda(|k|)$ in the neighborhood of $|k|=0$, has then been proposed in [3], where the interface operators are given by

$$
S_{B}=S_{T}=-i \omega \beta\left(I+\frac{\alpha}{\omega^{2}} \overrightarrow{\operatorname{curl}}_{\Gamma} \operatorname{curl} \Gamma_{\Gamma}\right)
$$

Taking $\alpha=-1$ this interface condition coincides with our symmetric interface conditions for $p=i \frac{\beta}{\omega}$. If $\beta \in \mathbb{R}$ then $\mathcal{R} e p=0$ and we have from Section 2.1 that $\rho=1$ for evanescent modes.

Notice however that the authors assume $\sqrt{\omega^{2}-|k|^{2}} \sim \omega$, which amounts to take into account, at once, only propagative modes, and, among them, only the ones with very small space frequency. Our approach differs a fortiori from this latter one, since we do not impose any restriction on the spatial frequency $|k|^{2}$.

\section{Numerical experiments}

In Figure 4 we compare the convergence history of the iterative procedure with the following interface conditions: optimized symmetric (i.e. (7) with $p_{T}=p_{B}$ given by (10)), optimized unsymmetric (i.e. (7) with $p_{B}=r$ and $p_{T}=i q, r$ given in (17) and $q$ given in (18)), the interface condition (24) and the one introduced by P. Chevalier (25) with $\zeta=i / 2 \omega$. We apply 
them to the model problem (23) and also to a similar one with a radiation condition on the whole boundary

$$
\begin{cases}\operatorname{curl} \operatorname{curl} \mathbf{u}-\omega^{2} \mathbf{u}=\mathbf{F} & \text { in } \Omega=[0,2] \times[0,1] \times[0,1] \\ \operatorname{curl} \mathbf{u} \times \mathbf{n}+i \omega \mathbf{u}_{\tau}=\mathbf{0} & \text { on } \partial \Omega\end{cases}
$$
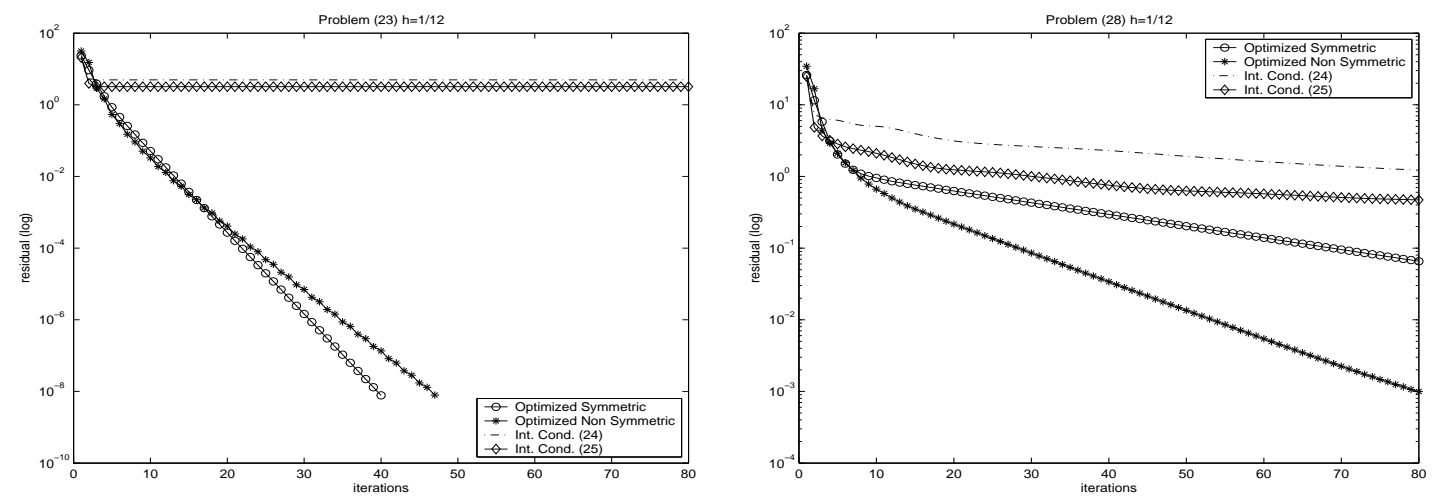

Figure 4: Convergence history for different interface conditions. Problem (23) (left) and Problem (28) (right): Iterative algorithm.

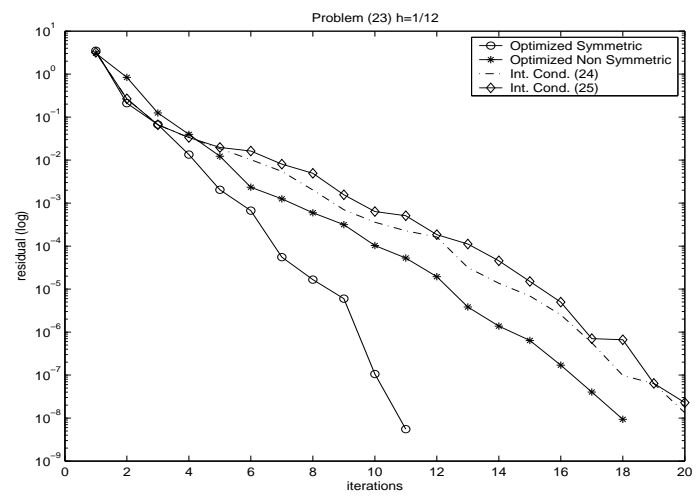

Figure 5: Convergence history for different interface conditions, Problem (23): Krylov algorithm.

We plot the residual for the first 80 iterations since its norm is greater that $10^{-8}$. For the model problem (23) the interface conditions (24) and (25) do not converge (note that the boundary condition in (23) differs from the one considered in [5]). For the model problem (28) the convergence of the four algorithms is very slow. In this case the fastest one is the one that uses the optimized unsymmetric interface conditions.

Finally, in Figure 5 we compare the convergence histories for the different interface conditions when the Krylov algorithm is applied to problem (23). Notice that none of the interface conditions (24) and (25) has been previously used in such a framework. All algorithms converge, and the ones with optimized conditions are faster. In this case the fastest one is the one that uses the optimized symmetric interface conditions. 


\section{Conclusions}

We proposed and analyzed two families of second order interface conditions to be used in the framework of non-overlapping domain decomposition methods for the time-harmonic Maxwell equations. These conditions have been derived by means of a Fourier analysis: the first family depends on a complex parameter while the second family depends on two real parameters. These parameters have been optimized, by minimizing the convergence rate of the method. The resulting interface operators have been applied to two different algorithms in the case of a two domains decomposition: the first algorithm is an iterative procedure of fixed point type and the second one is a Krylov subspace method. The numerical solution of some test problems has been addressed in order to validate the two algorithms: in both cases we obtained a convergent method. In particular, the Krylov subspace procedure, based on GMRES iterations, appears to be very efficient. Finally, we compared these optimized interface conditions with other conditions proposed in literature: evidence is that the optimized conditions provide more efficient and robust algorithms.

\section{References}

[1] A. Bossavit, Computational Electromagnetism. Variational Formulation, Complementarity, Edge Elements, vol. 2 of Electromagnetism Series, Academic Press, San Diego, CA, 1998.

[2] P. Chevalier, Méthodes numériques pour les tubes hyperfréquences. Résolution par décomposition de domaine, PhD thesis, Université Paris VI, 1998.

[3] P. Collino, G. Delbue, P. Joly, and A. Piacentini, A new interface condition in the non-overlapping domain decomposition for the Maxwell equations, Comput. Methods Appl. Mech. Engrg., 148 (1997), pp. 195-207.

[4] B. Després, Domain decomposition method and the Helmholtz problem, in First International Conference on Mathematical and Numerical Aspects of Wave Propagation (Strasbourg, 1991), Philadelphia, PA, 1991, SIAM, pp. 44-52.

[5] B. Després, P. Joly, And J. E. Roberts, A domain decomposition method for the harmonic Maxwell equations, in Iterative methods in linear algebra (Brussels, 1991), Amsterdam, 1992, North-Holland, pp. 475-484.

[6] M. J. Gander, F. Magoulès, And F. NATAF, Optimized schwarz methods without overlap for the Helmholtz equation, SIAM J. Sci. Comput., 24 (2002), pp. 38-60.

[7] P.-L. LIONs, On the Schwarz alternating method. III: a variant for nonoverlapping subdomains, in Third International Symposium on Domain Decomposition Methods for Partial Differential Equations, held in Houston, Texas, March 20-22, 1989, T. F. Chan, R. Glowinski, J. Périaux, and O. Widlund, eds., Philadelphia, PA, 1990, SIAM.

[8] P. Monk, Finite Element Methods for Maxwell's Equations, Oxford University Press, Oxford, 2003.

[9] J. NÉDÉLEC, Mixed finite elements in $R^{3}$, Numer. Math., 35 (1980), pp. 315-341.

[10] J. NÉDÉLEC, Eléments finis mixtes incompressibles pour l'équation des Stokes dans $\mathbb{R}^{3}$, Numer. Math., 39 (1982), pp. 97-112.

[11] J.-C. NÉdÉLEC, Acoustic and Electromagnetic Equations, Springer, New York, 2001.

[12] A. Quarteroni and A. Valli, Domain Decompostion Methods for Partial Differential Equations, Oxford University Press, 1999.

[13] H. VAn Der Vorst And J. Melissen, A Petrov-Galerkin type method for solving Ax $=b$, where $A$ is symmetric complex, IEEE Trans. Mag., 26 (1990), pp. 706-708. 